\title{
Clinicopathological features of Epstein-Barr virus infection, microsatellite instability, tumor mutation burden and PD-L1 status in Chinese patients with gastric cancer
}

\author{
Li Zhang \\ Peking University Cancer Hospital \\ Aiwen Wu \\ Peking University Cancer Hospital \\ Zhongwu Li ( $\sim$ zhongwulipku@163.com )
}

\section{Research}

Keywords: Gastric cancer, MSI, next generation sequencing, TMB, EBV-encoded RNA, PD-L1

Posted Date: September 24th, 2020

DOl: https://doi.org/10.21203/rs.3.rs-48875/v2

License: (c) (i) This work is licensed under a Creative Commons Attribution 4.0 International License.

Read Full License

Version of Record: A version of this preprint was published at Diagnostic Pathology on May 1st, 2021. See the published version at https://doi.org/10.1186/s13000-021-01099-y. 


\section{Abstract}

Objectives: Gastric cancer (GC) is the 4th most common type of cancer worldwide. Different GC subtypes exhibit unique molecular features that may potentially guide therapeutic decisions. The aim of the present study was to investigate Epstein-Barr virus (EBV) infection, microsatellite instability (MSI) status, the expression of programmed death-ligand 1 (PD-L1) and gene mutations in patients with surgicallytreated GC.

Methods: The data of 2,504 GC patients, who underwent potentially curative gastrectomy with lymphadenectomy at Peking University Cancer Hospital between 2013 and 2018, were reviewed from a prospectively collected medical database. We also analyzed the clinicopathological factors associated with the immunohistochemistry (IHC) profiles of these patients, and genetic alterations were analyzed using next generation sequencing (NGS).

Results: Mismatch repair-deficient (d-MMR) GC patients were found to have a higher probability of expressing PD-L1 $(p<0.001$, PD-L1 cutoff value $=1 \%)$. In addition, 4 and $6.9 \%$ of the 2,504 gastric cancer patients were EBV-positive and d-MMR, respectively. The number of MLH1/PMS2-negative cases was 126 (6\%), and the number of MSH2/MSH6-negative cases was $14(0.9 \%)$. d-MMR status was associated with a diffuse/mixed group ( $p<0.05$ ), but not with tumor differentiation. Furthermore, MSI and d-MMR GC status (detected by NGS and IHC, respectively) were consistently high, and the rate of MSI was higher in patients with d-MMR GC. A number of genes associated with DNA damage repair were detected in GC patients with MSI, including POLE, ETV6, BRCA and RNF43. In patients with a high tumor mutation burden, the most significantly mutated genes were LRP1B (79.07\%), ARID1A (74.42\%), RNF43 (69.77\%), ZFHX3 (65.12\%), TP53 (58.14\%), GANS (51.16\%), BRCA2 (51.16\%), PIK3CA (51.16\%), NOTCH1 (51.16\%), SMARCA4 (48.84\%), ATR (46.51\%), POLE (41.86\%) and ATM (39.53\%).

Conclusions: Using IHC and NGS, MSI status, protein expression, TMB and genetic alterations were identified in patients with GC, which provides a theoretical basis for the future clinical treatment of GC.

\section{Introduction}

Gastric cancer (GC) is the 4th most common cancer and the 2 nd leading cause of cancer-related death worldwide [1]. Comprehensive molecular characterization at the genomic and transcriptomic levels has led to the identification of distinct GC subtypes [2,3]. These different subtypes exhibit unique molecular features that could potentially be used to guide therapeutic decisions, and have been shown to have prognostic significance; for example, Epstein-Barr virus (EBV) infection has been associated with improved prognosis. Since the molecular classification of GC has potential prognostic and therapeutic implications, it may be used identify biomarkers and therapeutic targets for each subtype, particularly through stratification according to EBV infection and microsatellite instability (MSI) [4-8]. Currently, the expression of programmed death-ligand 1 (PD-L1) in tumor cells is a validated predictive marker for the tumor response to anti programmed cell death-1 protein (anti-PD-1) or PD-L1 immunotherapy in different 
malignancies, including GC [9]. According to a study of The Cancer Genome Atlas (TCGA), as well as recent clinical trials, EBV and MSI GC subgroups may benefit from therapy with PD-1/PD-L1 antibodies $[10,11]$. Furthermore, PD-1/PD-L1 inhibitors appear to enhance antitumor activity in patients with advanced GC [12-15]. However, the frequency and prognostic value of PD-L1 expression in GC remain controversial. Human epidermal growth factor receptor-2 (HER-2) is a 185-kDa transmembrane tyrosine kinase receptor of the epidermal growth factor receptor family, which has been identified as a protooncogene. Growing evidence suggests that HER-2 is an important biomarker and key driver of GC tumorigenesis, which has been associated with cellular proliferation, apoptosis and differentiation. When combined with systemic cytotoxic chemotherapy, Trastuzumab is a therapeutic option for patients with advanced or metastatic HER-2-positive GC. Moreover, HER-2 upregulation or gene amplification are important predictive markers in GC $[16,17]$. The aim of the present study was to investigate EBV infection status using in situ hybridization (ISH), and to determine mismatch repair (MMR) status and PD-L1 expression using immunohistochemistry (IHC), in surgically treated GC patients. Additionally, we analyzed the clinicopathological and prognostic factors associated with these IHC profiles, and used next generation sequencing (NGS) technology to analyze the gene alterations, MMR deficient (d-MMR)/MMR proficient ( $p-M M R$ ) status, tumor mutation burden (TMB) data and MSI status of patients with GC.

\section{Materials And Methods}

\subsection{Patients and general information}

In the present study, we reviewed all gastric adenocarcinoma patients from a prospective collected medical database, who underwent potentially curative gastrectomy with lymphadenectomy at Peking University Cancer Hospital between 2013 and 2018. The inclusion criteria included: (1) A positive diagnosis of gastric adenocarcinoma; and (2) the availability of formalin-fixed paraffin-embedded tissue blocks. Surgical specimens were fixed in $10 \%$ buffered formalin, and the slices were evaluated according to a protocol from the College of Chinese Pathologists. In some cases, IHC was performed to detect cytokeratin, an indicator of lymph node micro-metastasis. GC TNM staging was conducted according to the 2014 edition of Pathology and Genetics of Tumours of the Digestive System (World Health Organization Classification of Tumours S.). The study was approved by the ethics committee of Peking University Cancer Hospital, and all patients provided written informed consent prior to surgery.

All paraffin-embedded specimens were cut into 4-um sections using a conventional histological technique, and then transferred to slides. IHC staining was performed using the Herceptest kit $^{\text {TM }}$ (Dako, Carpinteria, CA, USA) according to the manufacturer's protocol, with an automatic immunostainer (Dako). Staining intensity was evaluated using the 0 to $3+$ scale according to the test scoring criteria.

\subsection{Immunohistochemical evaluation of PD-L1 expression}

All tissue slices were evaluated by two pathologists. Specimens were scored based on the area of positively stained tumor cells or tumor-infiltrating immune cells as follows: 1 , Positive staining area $<1 \%$; 2 , from $1 \%$ to $<10 \%$ positive staining; 3 , from $10 \%$ to $<50 \%$ positive staining; or 4 , $\geq 50 \%$ positive staining. 
The primary antibody against PD-L1 (SP142) was purchased from Spring Bioscience (Pleasanton, CA, USA).

\subsection{Evaluation of MMR protein expression and EBV infection status by IHC and ISH}

Tumors were considered to have lost MLH1, MSH2, PMS2 or MSH6 expression only if there was a complete absence of nuclear staining in tumor cells; normal epithelial cells and lymphocytes were used as the internal controls. Tumors lacking MLH1, MSH2, PMS2 or MSH6 expression were considered to be $\mathrm{d}-\mathrm{MMR}$, while those that maintained expression of these markers were considered to be p-MMR (as long as the tumor cell nucleus was positively stained, and regardless of the percentage positivity). MMR protein expression was assessed by IHC using antibodies against the following: MLH1 (clone no. GM002); MSH2 (clone no. RED2); MSH6 (clone no. EP49); and PMS2 (clone no. EP51) (all Gene Tech Biotechnology Co., Ltd., Shanghai, China). The complete absence of protein expression $(0+$ in $100 \%$ of cells) was considered to indicate the loss of MMR, and thus d-MMR. An EBV-encoded RNA (EBER) ISH kit (OriGene Technologies, Inc., Beijing, China) was used to determine EBV infection status, per the manufacturer's protocol.

\subsection{TMB and gene mutation analysis}

NGS technology was used to detect the MSI status of the GC samples, including TMB and gene mutations (ChosenMed, Inc., Beijing, China). TMB was assessed using the NGS platform (Illumina sequencing platform, PE150) with a sequencing depth greater than 3500x. The candidate MSI loci were detected by identifying a sequence of 1-5 bases with mutations that had repeated at least 5 times in the Bam file. The MSI threshold was determined according to large data sets from the European Genomephenome Archive and TCGA panels: $<20 \%$ was considered to be microsatellite stable (MSS), $20-30 \%$ indicated MSI-L and $>30 \%$ was considered as MSI-H. Gene mutations were obtained using an assembly clustering algorithm, not by simple cutoff values; the detection limit of the tissue samples was $2 \%$. The variation in the normal samples was 'SNP', and the specific variation of the tumor samples was 'somatic mutation'.

\subsection{Immunohistochemical detection of HER-2 expression}

HER-2 is located in the cell membrane. Tissues were stained and scored according to the HER-2 Detection Guide for Gastric Cancer as follows: $0,<10 \%$ tumor cell membrane staining; $1+, \geq 10 \%$ tumor cell membrane staining, weak or faintly visible membrane staining, or only partial membrane staining; $2+$, $\geq 10 \%$ tumor cells with weak to moderate basal membrane, lateral membrane or complete membrane staining; and $3+, \geq 10 \%$ strong tumor cell basal membrane, lateral membrane or complete membrane staining.

\subsection{Statistical analysis}


Comparisons between categorical variables were conducted using the $\chi^{2}$ test or Fisher's exact test as appropriate. Differences in $p$-values $<0.05$ were considered to be statistically significant.

\section{Results}

\subsection{Association between PD-L1 expression and the clinicopathological features of GC}

PD-L1-positive cases were defined by the presence of membrane staining in least $1 \%$ of tumor cells or tumor-infiltrating immune cells. Accordingly, the proportion of PD-L1-positive cases accounted for $20.2 \%$ of the patients investigated. Tumor cell PD-L1 expression was identified in 11.6, 10.9 and $4 \%$ of cases, at different cut-off points, respectively $(1,10$, and $50 \%$, according to the positively-stained area of the cell membrane). d-MMR GC patients were found to be more likely to express PD-L1 than p-MMR patients $(p<0.001 ;$ PD-L1 cutoff value $=1 \%)($ Figure 1$)$.

\subsection{MMR protein expression status and clinicopathological features}

In total, 140 of the 2,031 cases (6.9\%) were d-MMR. The number of MLH1/PMS2 protein deficient cases was $126 / 2031$ (6.2\%), and the number of MSH2/MSH6 deficient patients was 14/2031 (0.7\%). d-MMR status was associated with diffuse/mixed group $(p<0.05)$, but not with tumor differentiation $(p=0.256)$ (Table 1).

\subsection{EBER ISH ratio in patients with GC}

Of the 2,504 patients investigated, 96 cases (4\%) were EBV-positive and 2,408 (96\%) were EBER-negative. EBER-positive patients were predominantly male, with diffused/mixed Lauren type and poorly differentiated tumors $(p<0.001)$ (Table 2, Figure 2). The level of PD-L1 expression was not significantly different between EBER-positive and EBER-negative patients ( $p=0.524)$.

\subsection{The association between HER-2 expression, MMR status and EBER status}

In the present study, the number of HER-2 $1+$ patients was $628 / 2504(25.1 \%)$, the number of those with HER-2 2+ staining was 313/2504 (12.5\%), and 102/2504 patients (4.1\%) were HER-2 3+. There were 1,461 patients without HER-2 protein expression, and the ratio of positive-to-negative expression was $58.3 \%$. HER-2 expression was not found to be associated with MMR or EBER status ( $p=0.129$ and $p=0.300$, Table 3 and 4 , respectively).

\subsection{Results of NGS, and comparison of NGS-MSI and IHC-MMR results in GC}

The MSI (detected by NGS) and IHC results of patient with d-MMR GC were highly consistent; patients with d-MMR status had higher MSI scores, while those with p-MMR GC possessed comparatively lower scores (Figure 3). A number of genes associated with DNA damage repair (DDR) were detected in MSI patients, such as ETV6, TP53, BRCA, ATR, FANCA, BARD1, POLE and RNF43 (Figure 4). In GC patients with a high TMB, the most significantly mutated genes were LRP1B (79.07\%), ARID1A (74.42\%), 
RNF43(69.77\%), ZFHX3(65.12\%), TP53(58.14\%), GANS (51.16\%), BRCA2(51.16\%), PIK3CA (51.16\%), NOTCH1 (51.16\%), SMARCA4 (48.84\%), ATR (46.51\%), POLE (41.86\%) and ATM (39.53\%) (Figure 5).

\section{Discussion}

$\mathrm{GC}$ is the fourth most common type of cancer worldwide. Due to late diagnosis, the disease is frequently inoperable, and often recurs following curative resection. For patients with advanced and/or unresectable cancer, systemic chemotherapy is generally prescribed as the primary therapeutic option [18].

Traditionally, GC classification has been based on histopathological and morphological features, which were first described in $1965[19,20]$. Unfortunately, classifications based on morphology are unable to identify molecular targets. Therefore, large scale molecular profiling via NGS has resulted in the emergence of different molecular-based classification systems, which may be exploited for therapeutic intervention. HER-2 has long been associated with cellular differentiation, proliferation and apoptosis, and when used in conjunction with systemic chemotherapy, Trastuzumab is the therapeutic choice for patients with late-stage or metastatic HER-2-positive GC. Moreover, HER-2 overexpression or gene amplification is an important predictive indicator in GC; however thus far, no large-scale studies on HER-2 expression in GC have been conducted in China. Wang et al [21] studied 135 patients with GC, where the expression rate of HER-2 protein was 39.3\%. In our study, 2,504 GC patients were analyzed, among whom positive cases patients with HER-2 protein (3+)] accounted for $4.1 \%$, and patients with HER-2 protein (2+) expression accounted for $12.5 \%$. HER-2 expression was not found to be associated with EBER or MMR status, nor was it related to MMR status. However, in our previous study of $>3,000$ cases of colorectal cancer, HER-2 3+ positive expression was found to be more prevalent in p-MMR patients.

PD-L1 can be used as a marker of immunotherapeutic success, which is of great significance in clinical treatment. Since both markers are important for the treatment of GC, the relationship between the expression of HER-2 and PD-L1 requires further in-depth investigation. Anti-PD-1/PD-L1 immunotherapy may be used to treat patients with HER-2-negative GC, which provides an alternative treatment option for these individuals.

A number of studies have demonstrated that GC patients with EBV infection comprise 9\% of all cases of $\mathrm{GC}$, and that EBV infection constitutes a distinct clinicopathological and molecular entity [22]. In the present study, EBV positivity was $4 \%$, and the EBV-positive patients were predominantly male, with a diffused/mixed Lauren type and poor tumor differentiation $(p<0.001)$. The EBV infection rate in our study was also lower than the global average. The Alaska Native (AN) population exhibit the highest incidence and mortality rates of GC in North America, with an EBV infection rate of $20 \%$, which is far greater than the global average of $10 \%$ [23]. Molecular markers of solid tumors were also detected in $85 \mathrm{GC}$ patients, and the mutation burden and number of somatic mutations was lower in tumors from AN patients; the most common mutation was TP53. In a Japanese study of 1,067 GC cases, the positive rate of EBV infection was 7.1\% [24], indicating that the EBV infection rate of GC differs between regions. Less well understood is the involvement of EBV in chronic gastric inflammation, though multiple studies have argued that EBV (similar to and together with Helicobacter pylori) is an early participant in the GC 
oncogenic process, where it promotes chronic inflammation and subsequently aggravates tissue damage. Through the involvement of various cellular processes and signaling pathways, EBV infection may also contribute to the malignant transformation of GC cells. In contrast to other GC subtypes, GC patients with EBV infection exhibited a number of distinct characteristics in the present study. With a PDL1 positivity rate $>1 \%$, there was no significant difference in the level of PD-L1 expression between EBVpositive and EBV-negative patients $(p=0.524)$. In a small case study [25], PD-L1 expression was significantly associated with EBV infection $(p<0.001)$. In our study of 2,504 patients, high expression levels of PD-L1 were more likely to occur in d-MMR patients $(p<0.001 ; \mathrm{PD}-\mathrm{L} 1$ cutoff value $=1 \%)$. In a study by Haron et al [26], a total of $60 \mathrm{GC}$ cases were retrieved. Microsatellite analysis identified $10 \mathrm{MSI}-$ positive cases $(16.7 \%)$, of which six $(10.3 \%)$ did not express MLH1 $(n=3)$ or MSH2 $(n=3)$ protein. In our study, the number of MLH1/PMS2 protein deficient cases was $126(6 \%)$, and the number of MSH2/MSH6 protein deficient cases was $14(0.9 \%)$. Furthermore, d-MMR GC patients were more likely to express PD-L1 $(p<0.001)$. We think that different types of PD-L1 antibodies, different tissue processing methods, and different systems for evaluating PD-L1 may result in a wide range of different expression rates.

EBV is a carcinogenic virus, and studies have shown that the expression levels of ARID1A and PIK3CA are closely associated with the depth of GC invasion. As such, PIK3CA mutations in EBV-associated GC are usually accompanied by ARID1A mutations [27]. In the present study, we detected a high frequency of ARID1A and PIK3CA mutations; thus in the future, we intend to investigate the relationship between ARID1A, PIK3CA and EBER, and to analyze the expression of these proteins in GC and adjacent normal tissues.

In the present study, a number of the GC cases were sequenced, and cluster analysis was performed to identify various differentially expressed genes therein. Cho et al [28] performed massive parallel sequencing of 381 cancer-related genes and compared the results with the clinicopathological findings of 330 patients with GC. The most significantly mutated genes were TP53 (54\%), ARID1A (23\%), CDH1 (22\%), PIK3CA (12\%), RNF43 (10\%) and KRAS (9\%). Yoon et al [29] identified 18,377 MS mutations of five or more repeat nucleotides in gene coding sequences and untranslated regions (UTRs), and discovered 139 individual genes whose expression was downregulated in association with UTR MS mutation. In our study, numerous DDR-associated genes were detected in d-MMR patients, including ETV6, TP53, BRCA, POLE and RNF43; the most significantly mutated genes in d-MMR patients were LRP1B (79.07\%), ARID1A (74.42\%), RNF43 (69.77\%), ZFHX3 (65.12\%), TP53 (58.14\%), GANS (51.16\%), BRCA2(51.16\%), PIK3CA (51.16\%), NOTCH1 (51.16\%), SMARCA4 (48.84\%), ATR (46.51\%), POLE (41.86\%) and ATM (39.53\%). We also identified that the mutation rate of LRP1B was high, reaching $79.07 \%$. LRP1B belongs to the lowdensity lipoprotein (LDL) receptor gene family. Due to the interaction between these receptors and their ligands, they play a wide range of roles in normal cell functioning and development [30]. The LRP1B gene is also a novel candidate tumor suppressor that is associated with immunotherapeutic success. It has been found that nearly $40 \%$ of non-small cell lung cancer cell lines are inactivated by LRP1B alterations at the gene and transcriptional levels [31]. LRP1B is similar to LRP1 of the LDL receptor family. It is capable of inhibiting tumor cell invasion and metastasis by antagonizing extracellular uPA system hydrolyzed protein, degrading the extracellular matrix and preventing cellular migration. In the future, we 
aim to determine whether the expression levels of proteins encoded in association with GC are altered. LRP1B mutations have also been associated with a high TMB and low patient survival rates, though the relationship between LRP1B mutations and survival in GC is not well understood.

ZFHX3 plays an important role in the biological clock, which if disrupted, may be detrimental to human health. Various studies have shown that ZFHX3 inhibits the proliferation of prostate cancer cells by downregulating MYC gene expression [32], hence when mutated, ZFHX3 may influence the occurrence of cancer. RNF43 mutation results in a frame shift that leads to the early truncation and potential inactivation of the associated protein, and as such, may be a predictor of pathogenesis. Preclinical studies of gastric and colorectal cancer have shown that the inactivation of RNF43 promotes cellular proliferation and tumor growth [33]. Yu et al discovered a high frequency of RNF43 mutations in colorectal signet ring cell carcinoma, and that mutated RNF43 activates the Wnt pathway [34]. As with RNF43, frame shift mutations in the BRCA2 gene lead to the early truncation of the protein, and its subsequent inactivation may predict pathogenesis. BRCA2 mutations have been widely reported in breast cancer [35], but have not been extensively studied in GC. The PIK3CA Y1021C mutation is located within the PI3K/PI4K domain of the PIK3CA protein, which leads to an increase in the transformation ability of cultured cell lines [36].

GNAS R201C is located in the GTP binding region of the GNAS protein. In a mouse model, R201C resulted in the loss of GTP enzyme activity, the continuous activation of downstream signals, cellular proliferation and tumor formation. Studies have also shown that the mutation rate of GNAS in non-ampullary duodenal adenocarcinoma is 6.5\% [37]. However, GNAS mutations have been more extensively studied in tumors of the pancreatic and biliary system than in GC.

ATM mutation leads to premature truncation of the ATM protein. Due to the deletion of all known functional domains, predictive mutations result in the loss of protein function [38]. ARID1A mutations frequently occur in GC and are associated with poor patient prognosis, potentially because the AKT signaling pathway can be activated by the decreased expression or function of ARID1A. The levels of multiple immune markers and TMB in patients with ARID1A mutations were significantly higher than those in ARID1A wild-type patients. ARID1A promotes MMR, and as such, ARID1A defects are associated with MMR and MSI. The expression of PD-L1 in alimentary tract cancer patients with ARID1A mutations was significantly higher than that in wild-type patients [39-41]. In the present study results, NGS revealed a high number of ARID1A mutations in d-MMR patients, thus we intend to analyze the relationship between PD-L1 expression and ARID1A as a future research prospect. ARID1A is a subunit of the SWI/SNF chromatin remodeling complex. The E157G mutation is located in the phosphatase tensin type domain of PTEN, and has been predicted to result in the loss of protein function. Furthermore, V158F has been predicted to lead to PTEN inactivation. In patients with HER-2-positive GC, PTEN deletion mutations are associated with Trastuzumab resistance, and the loss of heterozygosis of this gene has been reported more frequently in GC [42]. The relationship between PTEN protein deletion and various gene alterations in $\mathrm{GC}$ is not clear. Kim et al revealed that of 322 patients with advanced GC, the mutation rate of PTEN was $10.6 \%$, and that the deletion of PTEN function was associated with high MSI and EBV-positive status 
[43]. In solid tumor patients receiving immunotherapy, the median overall survival (OS) of patients with POLE/POLD1 mutations was significantly improved compared with that of non-carriers. Additionally, $26 \%$ of patients with POLE/POLD1 gene mutations also exhibited MSI-H status. After omitting these patients, OS in the mutant group remained improved; that is to say that in patients with MSS (who generally do not benefit from immunotherapy), the potential value of immunotherapy can still be determined according to POLE/POLD1 gene mutations. Multivariate analysis confirmed that POLE/POLD1 mutation may be used as a novel independent index to predict immunotherapeutic value [44].

To the best of our knowledge, our study is the largest to investigate the pathological characteristics of GC patients in China. Using IHC, ISH and NGS, the results of this study provide a deeper understanding of GC, including MSI status, HER-2 and PD-L1 expression, TMB and gene alterations in GC patients, which offer a theoretical basis for the future clinical treatment of GC. Our future studies will aim to elucidate the mechanisms by which these mutations impact the development of GC.

\section{Abbreviations}

GC: gastric cancer; MSIهMicrosatellite instability $₫ T M B$ : tumor mutation burden; PD-L1: programmed death-ligand 1囚ISH囚in situ hybridization; IHC: immunohistochemistry; DDR: DNA damage repair

\section{Declarations}

\section{Acknowledgements}

Not applicable.

\section{Competing interests}

The authors declare no conflict of interest

\section{Authors' contributions}

Li Zhang conceived the study and drafted the manuscript. Li Zhang, Aiwen Wu and Zhongwu Li acquired the data. All authors read and approved the manuscript.

\section{Funding}

No funding was received.

\section{Availability of data and materials}

Not applicable.

\section{Ethics approval and consent to participate}


The study was approved by Peking University Cancer Hospital ethics committee. People who participated in this research had complete clinical data. Signed informed consents were obtained from the patients and/or the guardians.

\section{Consent for publication}

Not applicable.

\section{References}

1هRebecca L.Siegel, Kimberly D.Miller, Ahmedin Jemal DVM. Cancer Statistics. 2020.

2هCancer Genome Atlas Research Network. Comprehensive molecular characterization of gastric adenocarcinoma. Nature 2014; 513: 202-209.

3囚 Oh SC, Sohn BH, Cheong JH, et al. Clinical and genomic landscape of gastric cancer with a mesenchymal phenotype. Nat Commun 2018; 9:1777.

4『Kang BW, Baek DW, Kang H, Baek JH, Kim JG. Novel Therapeutic Approaches for Epstein-Barr Virus Associated Gastric Cancer. Anticancer Res 2019; 39: 4003-4010.

5『Kim MY, Kruger AJ, Jeong JY, et al. Combination Therapy with a PI3K/mTOR Dual Inhibitor and Chloroquine Enhances Synergistic Apoptotic Cell Death in Epstein-Barr Virus-Infected Gastric Cancer Cells. Mol Cells 2019; 42: 448-459.

6खVerma R, Agarwal AK, Sakhuja P, Sharma PC. Microsatellite instability in mismatch repair and tumor suppressor genes and their expression profiling provide important targets for the development of biomarkers in gastric cancer. Gene 2019; 710:48-58.

7囚Ho SWT, Tan P. Dissection of gastric cancer heterogeneity for precision oncology. Cancer Sci 2019; 110: 3405-3414.

8هYoshida T, Yamaguchi T, Maekawa S, et al. Identification of early genetic changes in welldifferentiated intramucosal gastric carcinoma by target deep sequencing. Gastric Cancer 2019; 22: 742750 .

9『Koemans WJ, Chalabi M, van Sandick JW, van Dieren JM, Kodach LL. Beyond the PD-L1 horizon: In search for a good biomarker to predict success of immunotherapy in gastric and esophageal adenocarcinoma. Cancer Lett 2019; 442:279-286.

10区 Le DT, Durham JN, Smith KN, et al. Mismatch repair deficiency predicts response of solid tumors to PD-1 blockade. Science 2017; 357: 409-413. 
11囚Nakayama A, Abe H, Kunita A, et al. Viral loads correlate with upregulation of PD-L1 and worse patient prognosis in Epstein-Barr Virus-associated gastric carcinoma. PLoS One 2019; 14: e0211358.

$12 \varangle$ Wang BC, Zhang ZJ, Fu C, Wang C. Efficacy and safety of anti-PD-1/PD-L1 agents vs chemotherapy in patients with gastric or gastroesophageal junction cancer: a systematic review and meta-analysis. Medicine (Baltimore) 2019; 98: e18054.

13凶Wang F, Wei XL, Wang FH, et al. Safety, efficacy and tumor mutational burden as a biomarker of overall survival benefit in chemo-refractory gastric cancer treated with toripalimab, a PD-1 antibody in phase Ib/II clinical trial NCT02915432. Ann Oncol 2019; 30: 1479-1486.

14区Noh BJ, Kim JH, Eom DW. Prognostic Significance of Categorizing Gastric Carcinoma by PD-L1 Expression and Tumor Infiltrating Lymphocytes. Ann Clin Lab Sci 2018; 48: 695-706.

$15 \llbracket$ Kono K, Nakajima S, Mimura K. Current status of immune checkpoint inhibitors for gastric cancer. Gastric Cancer 2020; 23: 565-578.

16खKijima T, Arigami T, Uenosono Y, et al. Comparison of HER2 Status Before and After Trastuzumabbased Chemotherapy in Patients with Advanced Gastric Cancer. Anticancer Res 2020; 40:75-80.

17凶Wang DS, Liu ZX, Lu YX, et al. Liquid biopsies to track trastuzumab resistance in metastatic HER2positive gastric cancer. Gut 2018; 68:1152-1161.

18囚Maron SB, Alpert L, Kwak HA, et al. Targeted therapies for targeted populations: anti-EGFR treatment for EGFR-amplified gastroesophageal adenocarcinoma. Cancer Discov 2018; 8: 696-713.

19囚Lauren P. The two histological main types of gastric carcinoma: diffuse and so-called intestinal-type carcinoma. An attempt at a histo-clinical classification. Acta Pathol Microbiol Scand 1965; 64: 31- 49.

20खMa J, Shen H, Kapesa L, Zeng S. Lauren classification and individualized chemotherapy in gastric cancer. Oncol Lett 2016; 11: 2959-2964.

$21 \otimes$ Wang YK, Wang SN, Li YY, et al. Methods and significance of the combined detection of HER2 gene amplification and chemosensitivity in gastric cancer. Cancer Biomark 2018; 21: 439-447.

22ه Shinozaki-Ushiku A, Kunita A, Fukayama M. Update on Epstein-Barr virus and gastric cancer(review). Int J Oncol 2015; 46: 1421-1434.

23凶Holly A Martinson, Dominic Mallari, Christine Richter, et al. Molecular Classification of Gastric Cancer among Alaska Native People. Cancers (Basel) 2020; 12:198.

24『Ayaka Yanagi,Jun Nishikawa, Kanami Shimokuri, Takuya Shuto,Tatsuya Takagi,Fumiya Takagi.Clinicopathologic Characteristics of Epstein-Barr Virus-Associated Gastric Cancer Over the Past Decade in Japan. Microorganisms. 2019; 7: 305. 
$25 \llbracket$ Nakayama A, Abe H, Kunita A, et al. Viral loads correlate with upregulation of PD-L1 and worse patient prognosis in Epstein-Barr Virus-associated gastric carcinoma. PLoS One 2019; 14: e0211358.

26冈Haron NH, Mohamad Hanif EA, Abdul Manaf MR, et al. Microsatellite Instability and Altered Expressions of MLH1 and MSH2 in Gastric Cancer. Asian Pac J Cancer Prev 2019; 20: 509-517.

27区Huan Zhou, Shun Tan, Hong Li, Xiangtao Lin. Expression and significance of EBV, ARID1A and PIK3CA in gastric carcinoma.Med Rep 2019;19:2125-2136.

28खCho J, Ahn S, Son DS, et al. Bridging genomics and phenomics of gastric carcinoma. Int J Cancer 2019; 145: 2407-2417.

29هYoon K, Lee S, Han TS, et al. Comprehensive genome- and transcriptome-wide analyses of mutations associated with microsatellite instability in Korean gastric cancers. Genome Res 2013; 23:1109-1117.

30冈C X Liu, Y Li, L M Obermoeller-McCormick, A L Schwartz, G Bu. The putative tumor suppressor LRP1B, a novel member of the low density lipoprotein (LDL) receptor family, exhibits both overlapping and distinct properties with the LDL receptor-related protein. J Biol Chem. 2001; 3:276:28889-28896.

31区Chen $\mathrm{H}$, Chong W, Wu Q, Yao Y, Mao M, Wang X. Association of LRP1B Mutation with Tumor Mutation Burden and Outcomes in Melanoma and Non-small Cell Lung Cancer Patients Treated With Immune Check-Point Blockades. Front Immunol. 2019; 21;10:1113.

32هHu Q, Zhang $B$, Chen $R$, et al. ZFHX3 is indispensable for ER $\beta$ to inhibit cell proliferation via MYC downregulation in prostate cancer cells. Oncogenesis 2019;12;8:28.

33凶McCarthy AJ, Serra S, Chetty R. Traditional serrated adenoma: an overview of pathology and emphasis on molecular pathogenesis. BMJ Open Gastroenterol 2019; 6: e000317.

34囚Yaqi Li, Jian Li, Renjie Wang, Long Zhang Guoxiang Fu, Fa-Xing Yu. Frequent RNF43 mutation contributes to moderate activation of Wnt signaling in colorectal signet-ring cell carcinoma. Protein Cell. 2020; 11: 292-298.

35『Narod SA, Foulkes WD. BRCA1 and BRCA2: 1994 and beyond. Nat Rev Cancer. 2004; 4:665-676.

36冈 Jung EJ, Suh JH, Kim WH, Kim HS. Clinical significance of PI3K/Akt/mTOR signaling in gastric carcinoma. Int J Clin Exp Pathol. 2020; 13:995-1007.

37囚Jiro Watari, Seiichiro Mitani, Chiyomi Ito, Katsuyuki Tozawa, Toshihiko Tomita, Hiroto Miwa.Molecular alterations and PD-L1 expression in non-ampullary duodenal adenocarcinoma: Associations among clinicopathological, immunophenotypic and molecular features. Sci Rep. 2019; 9:10526. 
38 $₫$ Shira Peleg Hasson, Tehillah Menes, and Amir Sonnenblick. Comparison of Patient Susceptibility Genes Across Breast Cancer: Implications for Prognosis and Therapeutic Outcomes. Pharmgenomics Pers Med. 2020; 13: 227-238.

39囚Ober JM, Halske C, Behrens HM, Kruger S, Rocken C. Intratumoral heterogeneity and loss of ARID1A expression in gastric cancer correlates with increased PD-L1 expression in Western patients. Hum Pathol 2019; 94: 98-109.

40区 Hu GY, Tu W, Yang L, Peng G, Yang L. ARID1A deficiency and immune checkpoint blockade therapy: From mechanisms to clinical application. Cancer Lett 2020; 31:148-155.

41هLi L, Li M, Jiang Z, Wang X. ARID1A Mutations Are Associated with Increased Immune Activity in Gastrointestinal Cancer. Cells 2019;8: E678.

42区Cetintas VB, Batada NN. Is there a causal link between PTEN deficient tumors and immunosuppressive tumor microenvironment? J Transl Med 2020; 18:45. doi: 10.1186/s12967-02002219-w.

43囚Binnari Kim, So Young Kang, Deokgeun Kim, You Jeong Heo, Kyoung-Mee Kim. PTEN Protein Loss and Loss-of-Function Mutations in Gastric Cancers: The Relationship with Microsatellite Instability, EBV, HER2, and PD-L1 Expression囚cancers (Basel)2020;12(7):1724.

44囚Feng Wang, Qi Zhao, Rui-Hua Xu, et al. Evaluation of POLE/POLD1 Mutations as Biomarkers for Immunotherapy Outcomes Across Multiple Cancer Types. JAMA Oncol 2019; 5: 1504-1506.

\section{Tables}

Table1 PD-L1 expression, EBER ISH status withclinic-pathological features of GC patients 


\begin{tabular}{|c|c|c|c|}
\hline Variables & EBER ISH status & & $p$ value \\
\hline & EBER positive $(+)$ & EBER negative (-) & \\
\hline Sex & & & $0.003^{*}$ \\
\hline male & 82 & 1717 & \\
\hline female & 14 & 691 & \\
\hline Lauren type & & & $0.000 *$ \\
\hline Diffuse/mixed & 77 & 1503 & \\
\hline intestinal & 19 & 905 & \\
\hline Differentiation & & & $0.001^{*}$ \\
\hline poorly differentiated & 79 & 1600 & \\
\hline well-moderately & 17 & 808 & \\
\hline T stage & & & 0.322 \\
\hline pT3+T4 & 55 & 1500 & \\
\hline $\mathrm{pT} 1+\mathrm{T} 2$ & 41 & 908 & \\
\hline Lymphnodemetastasis & & & 0.326 \\
\hline LNM+ & 53 & 1450 & \\
\hline LNM- & 43 & 958 & \\
\hline PD-L1 expression & & & 0.524 \\
\hline PD-L1 - & 52 & 1617 & \\
\hline PD-L1>=1\% & 25 & 664 & \\
\hline
\end{tabular}

Table2 Clinicopathological features of 2504p-MMR and d-MMR GC patients 


\begin{tabular}{|c|c|c|c|}
\hline Variables & MMR status & & $p$ value \\
\hline & $\mathrm{p}-\mathrm{MMR}$ & d-MMR & \\
\hline Sex & & & 0.102 \\
\hline male & 516 & 45 & \\
\hline female & 1374 & 86 & \\
\hline Lauren type & & & $0.012^{\star}$ \\
\hline Diffuse/mixed & 1201 & 74 & \\
\hline intestinal & 690 & 66 & \\
\hline Differentiation & & & 0.256 \\
\hline poorly & 1264 & 87 & \\
\hline well-moderately & 627 & 53 & \\
\hline T stage & & & 0.038 \\
\hline pT3+T4 & 1193 & 88 & \\
\hline $\mathrm{pT} 1+\mathrm{T} 2$ & 698 & 52 & \\
\hline Lymphnode metastasis & & & 0.246 \\
\hline LNM+ & 1121 & 76 & \\
\hline LNM- & 770 & 64 & \\
\hline PD-L1 expression & & & $0.000 *$ \\
\hline PD-L1 - & 1337 & 69 & \\
\hline PD-L1>=1\% & 554 & 71 & \\
\hline
\end{tabular}

Table 3 Correlation of HER-2 expression and EBER statusin GC patients

\begin{tabular}{|llll|}
\hline Variables & EBER+ & EBER- & $p$ value \\
\hline HER-2 3+ & 2 & 102 & 0.300 \\
\hline HER-2 0/1+/2+ & 94 & 3205 & \\
\hline
\end{tabular}

Table 4 Correlationof HER-2 expression and MMR status in 2504 GC patients 


\begin{tabular}{|llll|}
\hline Variables & p-MMR & d-MMR & $p$ value \\
\hline HER-2 3+ & 75 & 2 & 0.129 \\
\hline HER-2 0/1+/2+ & 1816 & 138 & \\
\hline
\end{tabular}

\section{Figures}
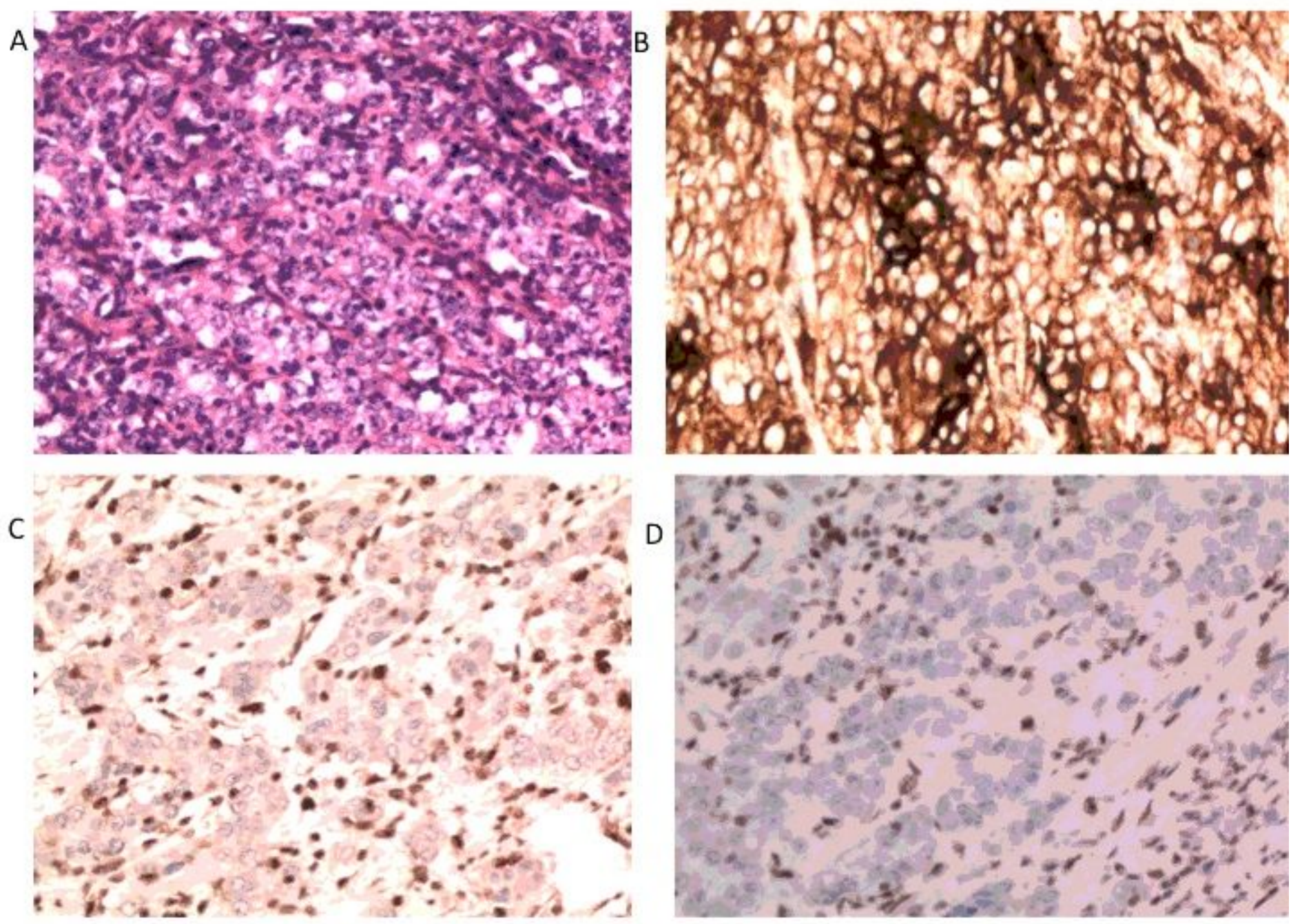

Figure 1

PD-L1 strong staining ind-MMR gastric cancer patients. A.poorly differentiated gastric cancer, Hematoxylin and Eosin staining(H\&E staining), 200x magnification; B.The positive staining area of PD-L1 on tumor cells was more than $90 \%$, moderate to strong positive, 200x magnification; C.MLH1 IHCnegative staining, 200x magnification,stromal cells with positive staining as internal control; D. PMS2 IHCnegative staining, 200x magnification, stromal cells with positive staining as internal control. 

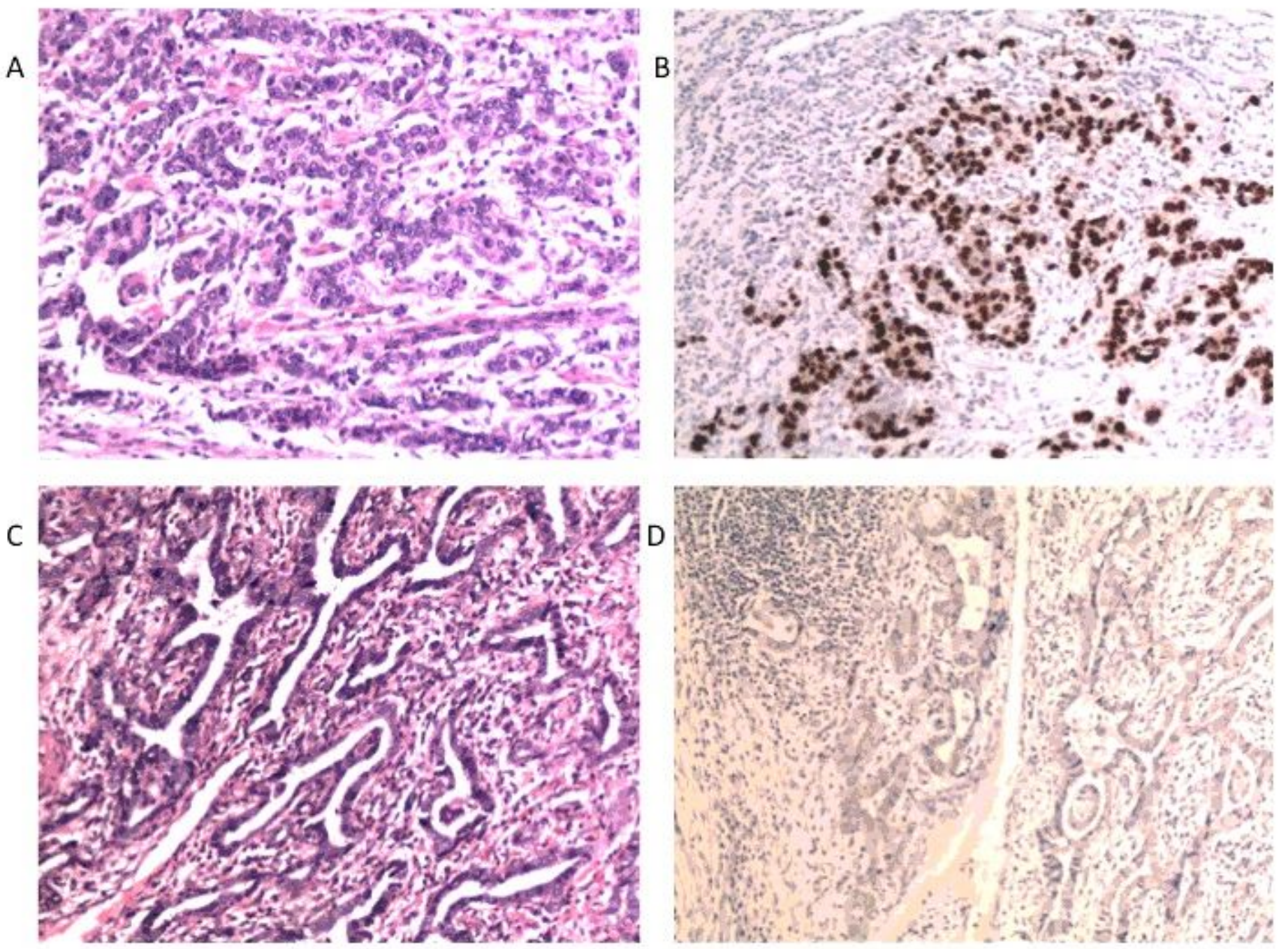

Figure 2

EBERpositive patients were usually had diffused/mixed Lauren type and poor differentiation. A.poorly differentiated gastric cancer, diffuse type, H\&Estaining, 200x magnification; B.poorly differentiated gastric cancer, EBER ISH positive staining, 200x magnification C. moderately differentiated gastric cancer, intestinal type, H\&E staining, 200x magnification; D. moderately differentiated gastric cancer,EBER ISH negative staining, 200x magnification; 


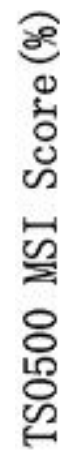

옹

$50-$

0 -

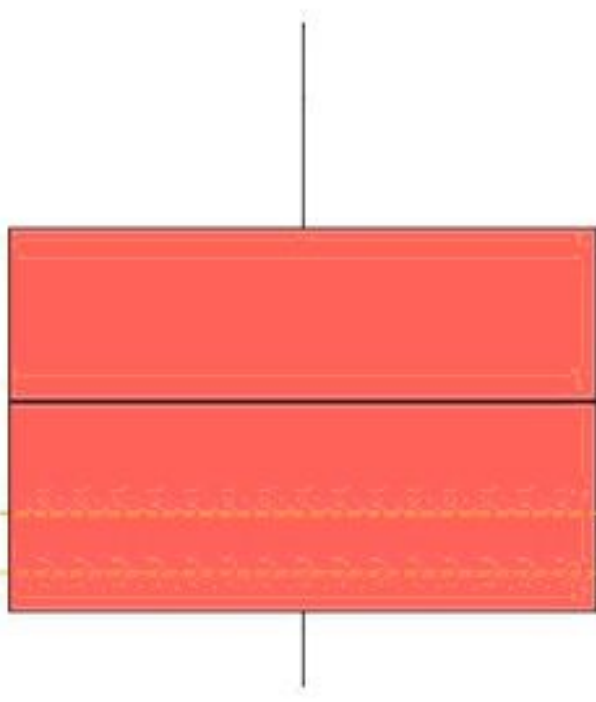

dMMR

$(n=43)$

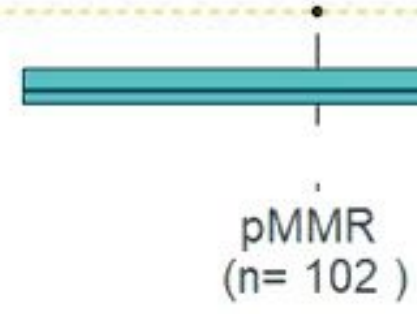

Figure 3

The results of MSI detected by NGSwere highly consistent with IHC results of d-MMR GC. d-MMR patients had higher MSI scores. 


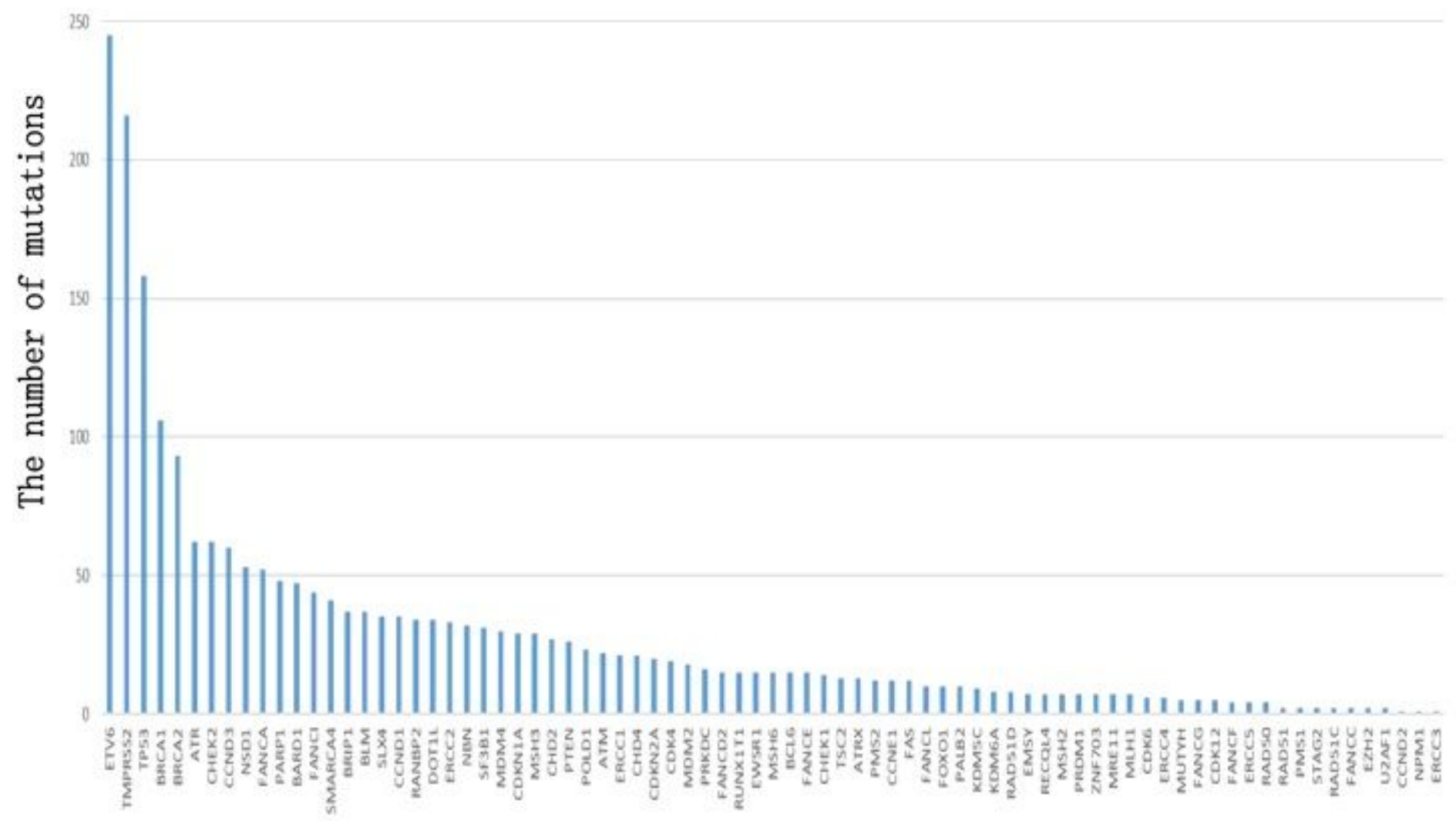

The most mutated gene in d-MMR gastric cancer

Figure 4

NGS results of d-MMR gastric cancer patients. Many pathogenic genes were detected, such as POLE, ETV6, TMPRS52,BRCA1,BRCA2,TP53and other genes. 


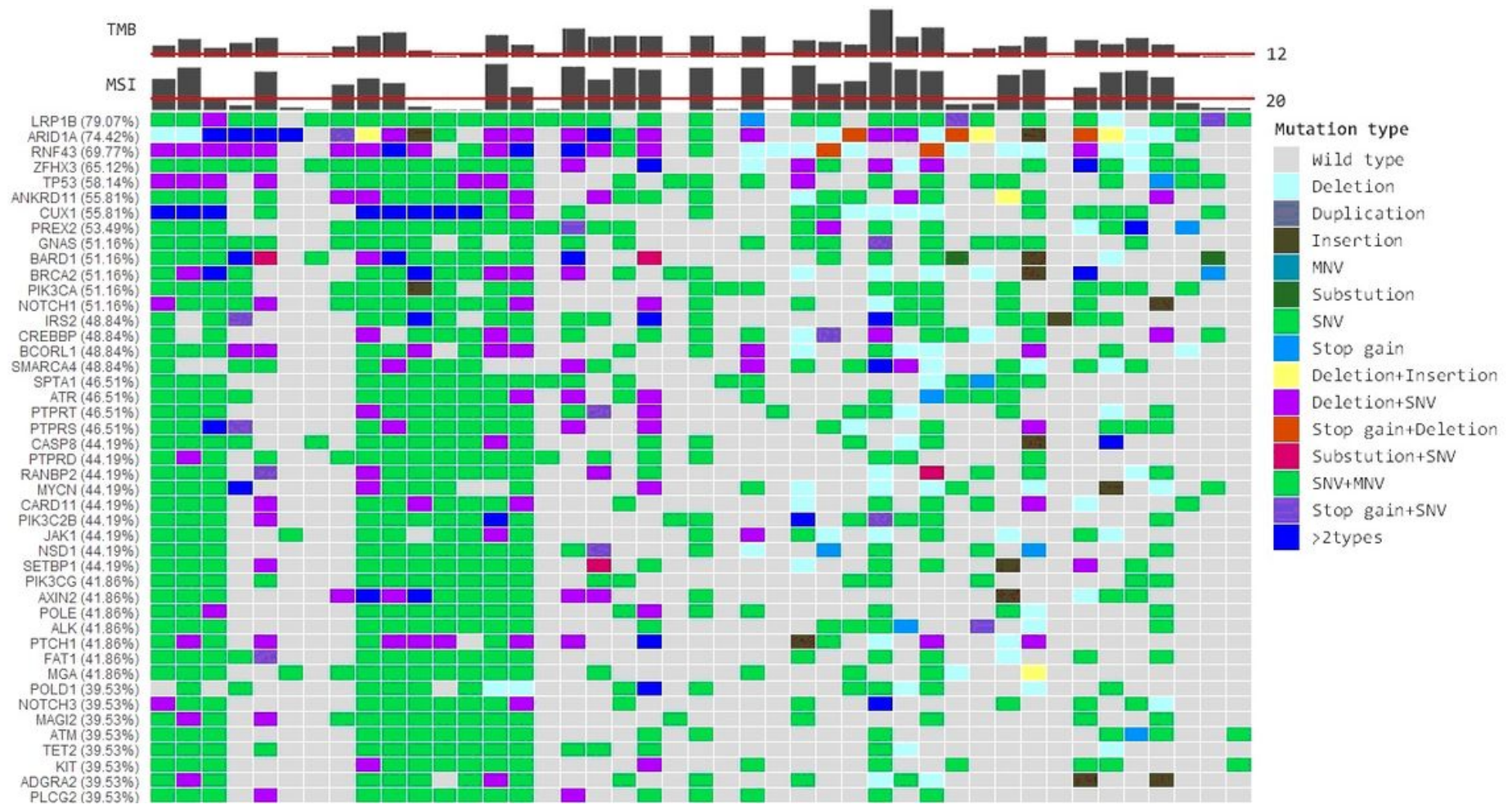

Figure 5

TMB $\triangle \mathrm{MSI}$ status, gene mutation frequency and mutation type detected by NGS. In high TMB and MSI tumors, we can see the deletion mutation of some genes,such as RNF43,BCORL1,ATR etc. 